\title{
SUPERNOVA IMPLICATIONS FOR THE MAGNETIC TRANSITION MOMENT OF MAJORANA NEUTRINOS
}

\author{
Miriam LEURER and Jiang LIU \\ The Randall Laboratory of Physics, University of Michigan, Ann Arbor, MI 48109-1120, USA
}

Received 9 December 1988

\begin{abstract}
A large magnetic moment or transition moment of the neutrino have been suggested as explanations for the solar neutrino puzzle. These scenarios have the attractive feature of incorporating an anticorrelation of the solar neutrino flux with sun spot activity. Recently it has been claimed that the 1987 supernova results put a severe bound on the neutrino magnetic moment, allowing only values that are too small to account for the solar neutrino flux depletion. Here we show that this bound does not apply to the transition magnetic moment of Majorana neutrinos. Other laboratory, astrophysical and cosmological bounds are also discussed.
\end{abstract}

Two years ago Voloshin, Vysotsky and Okun (VVO) [1] revived and elaborated on a solution to the solar neutrino puzzle first suggested by Cisneros [2]. According to refs. [1,2] the neutrino is of Dirac type and has a large magnetic moment. The lefthanded neutrino $\left(v_{\mathrm{e}}\right)_{\mathrm{L}}$ undergoes spin precession in the strong magnetic fields present in the sun and so emerges as a sterile, undetectable $\left(v_{e}\right)_{R}$. VVO were motivated by the observation [3] that the solar neutrino flux seems to be anticorrelated with sun spot activity. Accordingly, they suggested that spin precession takes place in the convective zone of the sun. Assuming the magnetic fields in the convective zone to be as large as several kilogauss and the width of the zone to be $2 \times 10^{5} \mathrm{~km}$, VVO required the magnetic moment $\mu$ to be $(1-10) \times 10^{-11} \mu_{\mathrm{B}}$ (where $\mu_{\mathrm{B}}$ is the Bohr magneton $e / 2 m_{\mathrm{e}}$ ). Such a magnetic moment is consistent with present laboratory bounds [4]. The lower VVO value $\mu \sim 10^{-11} \mu_{\mathrm{B}}$ is consistent also with a cosmological bound derived by Morgan [5] and with an astrophysical bound discussed in ref. [6]. However, as was pointed out by several authors [7], the observation of the neutrino burst from SN $1987 \mathrm{~A}$ implies that $\mu \leqslant 10^{-12} \mu_{\mathrm{B}}$. This bound is one order of magnitude lower than the minimal magnetic moment required by VVO.

In this note we wish to point out that another proposed solution to the solar neutrino puzzle, which also incorporates anticorrelation of the flux with sun spot activity, is consistent with all laboratory, cosmological and astrophysical bounds. The solution we refer to was proposed by Lim and Marciano [8] and by Akhmedov [9] (LMA). Among other possibilities, they suggest that neutrinos are Majorana particles with magnetic transition moments. (CPT invariance forbids diagonal magnetic moments of Majorana neutrinos but allows off-diagonal, flavour changing, transition moments). According to LMA, the solar $\left(v_{\mathrm{c}}\right)_{\mathrm{L}}$ is rotated into a $\left(\bar{v}_{\mu}\right)_{\mathrm{R}}$ in an MSW [10,11] fashion: The mass squared difference is such that in vacuum $\left(\bar{v}_{\mu}\right)_{\mathrm{R}}$ is heavier than $\left(\mathrm{v}_{\mathrm{e}}\right)_{\mathrm{L}}$ but in the core of the sun $\left(v_{e}\right)_{L}$ is heavier, due to coherent weak interaction with matter. At the resonance region (where the mass difference changes sign) the interaction of the magnetic transition moment with the strong solar magnetic fields serves as the mixing term necessary to rotate $\left(v_{c}\right)_{L}$ into $\left(\bar{v}_{\mu}\right)_{R}$. The depletion of electron neutrino flux will be correlated with sun spot activity if the resonance occurs in the convective zone. To obtain such a correlation, LMA require:

$$
\begin{aligned}
& \Delta m^{2} \simeq 10^{-7}-10^{-8} \mathrm{eV}^{2} \text { and } \\
& \mu \simeq(1-10) \times 10^{-11} \mu_{\mathrm{B}} .
\end{aligned}
$$

The condition on $\Delta m^{2}$ is important for the location of the resonance (in the convective zone) and the 
condition on the magnetic transition moment $\mu$ determines the resonance adiabaticity (so that on the average, the $v_{\mathrm{e}}$ flux is suppressed by a factor of 3 ).

The crucial difference between the VVO and LMA scenarios, which makes the last withstand the implications of SN $1987 \mathrm{~A}$ is that, according to VVO, $\left(v_{\mathrm{c}}\right)_{\mathrm{L}}$ rotates to sterile $\left(v_{\mathrm{e}}\right)_{\mathrm{R}}$ while LMA propose that $\left(v_{\mathrm{e}}\right)_{\mathrm{L}}$ rotates to the weakly interacting $\left(\bar{v}_{\mu}\right)_{R}$. In the following we will review the bounds on the magnetic moment and discuss which of them apply to the LMA case. We will also discuss an additional cosmological bound relevant only to flavour changing magnetic transition moments. The final result is that the LMA solution is consistent with all these bounds.

A laboratory bound [4]. Study of the recoiling electron in elastic ve scattering implies: $\mu \leqslant 1.5 \times 10^{-10} \mu_{\mathrm{B}}$, where $\mu$ is the magnetic moment of a Dirac electron neutrino. This bound holds also for a magnetic transition moment involving Majorana $\left(v_{c}\right)_{L}$ and $\left(\bar{v}_{\mu}\right)_{R}$ if $\Delta m^{2}$ is as small as $10^{-7}-10^{-8} \mathrm{eV}^{2}$.

A cosmological bound [5]. Morgan pointed out that if neutrinos were Dirac particles with large magnetic moments then in the big bang, the additional righthanded degrees of freedom would be excited down to low temperatures. Helium synthesis would then be disrupted unless $\mu \leqslant 2 \times 10^{-11} \mu_{\mathrm{B}}$. This bound does not apply to the magnetic transition moment of Majorana neutrinos since no additional degrees of freedom are excited in this case.

An astrophysical bound [6]. A bound of $\mu \leqslant 1.1 \times 10^{-11} \mu_{\mathrm{B}}$ was obtained by observing that larger magnetic moments would lead to rapid cooling of degenerate dwarf stars through neutrino pair emission. This bound applies also to LMA magnetic moments.

The last two bounds allow only the lower $\mu$ value in both the VVO and LMA scenarios: $\mu \simeq 10^{-11} \mu_{\mathrm{B}}$. In the LMA case the $\Delta m^{2}$ values are linked to the $\mu$ values through the adiabaticity condition and so one finds that $\Delta m^{2}$ should be $\sim 10^{-8} \mathrm{eV}^{2}$.

The supernova bound. We start by a rough and brief review of the standard model (no magnetic moments or transition magnetic moments) [12]. v's and $\bar{v}$ 's are pair-produced in the hot supernova core (electron neutrinos are also produced in the neutronization process: $\left.p+e^{-} \rightarrow n+v_{e}\right)$. Being trapped in the core by weak scattering on the highly dense matter, the $v$ 's and $\bar{v}$ 's drift out slowly, keeping all the while in thermal equilibrium with their immediate neighbourhood. At the neutrino sphere, the density is considerably reduced and matter becomes transparent to neutrinos. From now on the neutrinos will stream out, interacting only coherently with matter (that is, the only matter effect is to change the neutrinos' effective mass, as was originally discussed by Wolfenstein [11]). Roughly, these thermal neutrinos carry away $90 \%$ of the collapse energy, and the energy flux is equally distributed [13] among the six species $\left(v_{\mathrm{e}}\right)_{\mathrm{L}}$, $\left(\bar{v}_{\mathrm{c}}\right)_{\mathrm{R}},\left(v_{\mu}\right)_{\mathrm{L}},\left(\bar{v}_{\mu}\right)_{\mathrm{R}}\left(v_{\tau}\right)_{\mathrm{L}},\left(\bar{v}_{\tau}\right)_{\mathrm{R}}$. The $v_{\mathrm{c}}$ 's (and $\bar{v}_{\mathrm{e}}$ 's) are trapped for a little longer than the $\mu$ and $\tau$ neutrinos due to their charged current interaction with electrons. Consequently, the $v_{\mathrm{c}}$ sphere is at somewhat bigger radius and lower temperature than the $v_{\mu}$ and $v_{\tau}$ sphere. It is estimated that the average $v_{c}$ energy is half the average of $\nu_{\mu}$ or $v_{\tau}$ energy and that the number of emitted $v_{\mathrm{e}}$ 's is twice the number of emitted $v_{\mu}$ 's or $v_{\tau}$ 's. Of the neutrinos emitted by $\mathrm{SN}$ 1987A we, on earth, could only expect to see the $\bar{v}_{\mathrm{e}}$ pulse. Its predicted characteristics are its duration, spectrum and intensity: Duration should be around $10 \mathrm{~s}$, reflecting the long time the neutrinos need to drift out of the core. The spectrum is expected to be concentrated around a few $\mathrm{MeV}$ reflecting the temperature of the $v_{\mathrm{e}}$ sphere. The intensity should correspond to about $15 \%$ of the collapse energy. Within the theoretical errors and the statistical limitations, all these features were indeed seen.

Following the authors of ref. [7] we now describe what would have happened if the $v_{\mathrm{e}}$ was of Dirac type and had a large magnetic moment. In this case another, quicker channel of cooling would open for the hot collapsing core: An $v_{\mathrm{e}}$, produced in the interior of the core will not have to go through the slow process of drifting to the neutrino sphere. Instead, it will scatter electromagnetically on an electron or a proton and will turn into a sterile $\left(v_{\mathrm{e}}\right)_{\mathrm{R}}$. The $\left(v_{\mathrm{e}}\right)_{\mathrm{R}}$, being indifferent to matter, will immediately emerge out of the core. Unless $\mu \leqslant 10^{-12} \mu_{\mathrm{B}}$, most of the collapse energy will be emitted in this way within $2 \mathrm{~s}$ or less. The neutrino pulse observed on earth in this case will strongly differ from the previously described standard model prediction: Its duration will be much shorter $(2 \mathrm{~s}$ or less, instead of $10 \mathrm{~s}$ ) and, in addition, either the spectrum or the intensity will be drastically modified: If the sterile neutrinos rotate back to weakly interacting ones then the spectrum of the observed pulse will be 
concentrated around $200 \mathrm{MeV}$ (instead of few $\mathrm{MeV}$ ), reflecting the high temperatures of the interior of the core. If the sterile neutrinos do not rotate back then the detectable pulse is considerably depleted: The measured intensity will correspond to far less than $15 \%$ of the collapse energy.

Voloshin [14] has suggested the possibility that the spectrum and the intensity will not be effected by $\mu \simeq 10=^{11} \mu_{\mathrm{B}}$. He pointed out that the sterile neutrinos, on their way out of the core, pass a resonance region where they rotate back to weakly interacting neutrinos. This resonance occurs inside the neutrino sphere. Once the neutrinos have passed the resonance and are weakly interacting again, they find themselves trapped. The neutrinos are now forced into thermalizing with their neighbourhood and when finally they emerge out of the neutrino sphere their average energy is only a few MeV. Voloshin's scenario may indeed solve the problems related to the spectrum and the intensity of the pulse. However, even in this case, the duration of the pulse is far shorter than $10 \mathrm{~s}$, since most of their way through the core the neutrinos were sterile and free.

As the results of SN 1987A [15] have confirmed the standard model predictions for the neutrino pulse, one must conclude that $\mu$ is smaller than $10^{-12} \mu_{\mathrm{B}}$. VVO solution for the solar neutrino puzzle seems therefore to be excluded. We should note, however, that in a recent preprint Barbieri, Mohapatra and Yanagida [16] suggest a way for VVO neutrinos to go around the supernova bound. They discuss a specific model [17] and show that the same nonstandard interactions that are responsible for a large magnetic moment, also lead to trapping of the righthanded neutrinos in the supernova core, thereby avoiding the fatal shortening of the thermal neutrino pulse.

Consider now the case of LMA Majorana neutrinos with a flavour-changing magnetic moment $\mu$. In the hot dense core of the supernova an electron neutrino $\left(v_{\mathrm{e}}\right)_{\mathrm{L}}$ may scatter electromagnetically into $\left(\bar{v}_{\mu}\right)_{R}$. Nothing much will happen as $\left(\bar{v}_{\mu}\right)_{R}$ is not sterile but weakly interacting and trapped in the core. We do not expect $\mu$ of the order of $10^{-11} \mu_{\mathrm{B}}$ to lead to any drastic change in the neutrino pulses emerging from the neutrino spheres. Let us now follow the neutrinos on their way from the neutrino sphere to earth.
The v's may pass through two LMA resonances $\left(v_{\mathrm{e}}\right)_{\mathrm{L}} \leftrightarrow\left(\bar{V}_{\mu}\right)_{\mathrm{R}}$ and they also pass through an MSW \#1 resonance $\left(v_{\mathrm{e}}\right)_{\mathrm{L}} \leftrightarrow\left(v_{\mu}\right)_{\mathrm{L}}$. Note that in the sun $V_{\mathrm{e}}$ 's pass through a single LMA resonance, while in the supernova they may pass through two of them, through one or through none. The reason is that resonance occurs at a point where the quantity $\Delta=N_{\mathrm{e}}-N_{\mathrm{n}}$ reaches a certain value $\Delta_{0}$ [8] ( $N_{\mathrm{e}}, N_{\mathrm{n}}$ are the number densities of electrons and neutrons respectively). In the sun $\Delta$ is monotonically decreasing from the center outward and it therefore passes the resonance value $\Delta_{0}$ once. In the supernova $\Delta$ starts in the neutron-rich and dense core at very negative values, it climbs up to positive values when it approaches the proton-rich Hydrogen shell and decays to zero as the matter density fades away out of the star. Depending on the maximum value of $\Delta$ in the supernova, it goes through the resonance value 0,1 or 2 times. The $\bar{v}_{c}$ 's of the supernova pass through an LMA resonance $\left(\left(\bar{v}_{\mathrm{e}}\right)_{\mathrm{R}} \leftrightarrow\left(v_{\mu}\right)_{\mathrm{L}}\right)$ once, when $\Delta=-\Delta_{0}$. In he sun $\Delta$ is positive and no such resonance occurs but, as was pointed out by Lim and Marciano [8], the case of the supernova is different due to the neutron-rich environment of its interior. The adiabaticity of the various resonances depends on the presently unknown supernova profiles of electron and neutron number densities, magnetic field and on the unknown $\nu_{\mathrm{e}}-v_{\mu}$ mixing. Out of the supernova star the intergalactic magnetic field $\left(\sim 10^{-6} \mathrm{G}\right)$ is too weak and its induced "spin precession" is quenched by the $v_{\mathrm{e}}-v_{\mu}$ mass difference. The final result of all this 163000 thousand years of history is that the $\left(\bar{v}_{e}\right)_{R}$ pulse that finally reaches terrestrial detectors is some mixture of the original $\left(\bar{v}_{\mathrm{c}}\right)_{\mathrm{R}}$ and $\left(v_{\mu}\right)_{\mathrm{L}}$ pulses that came out of the neutrino sphere. (The arriving $\left(V_{c}\right)_{L}$ pulse is a mixture of the original $\left(v_{c}\right)_{L},\left(\bar{v}_{\mu}\right)_{R},\left(v_{\mu}\right)_{L}$ and $\left(\bar{v}_{e}\right)_{R}$ pulses $)$. The whole effect of the magnetic transition moment amounts to a very mild modification in the spectrum and intensity of the detectable $\left(\bar{v}_{\mathrm{e}}\right)_{\mathrm{R}}$. Only factors of $\sim 2$ are involved in the pulse modification and in view of present theoretical uncertainties and of the low statistics of Kamiokande and IBM events we conclude that the LMA scenario with $\mu \sim 10^{-11} \mu_{\mathrm{B}}$ and $\Delta m^{2} \sim 10^{-8} \mathrm{eV}^{2}$ may not be excluded.

$\#$ The MSW resonance is expected to be nonadiabatic for $\Delta m^{2}=10^{-8}$, unless the mixing angle is large [18]. 
We would now like to comment on the "prompt neutronization neutrino burst" $\# 2$. According to the standard model a prompt $\left(v_{\mathrm{e}}\right)_{\mathrm{L}}$ pulse is released within a few milliseconds after collapse. This pulse carries $\sim 10 \%$ of the collapse energy, and its spectrum is concentrated around a few $\mathrm{MeV}$ (the prompt and thermal neutrinos carry away almost all of the collapse energy). The prompt neutrinos originate in the "neutronization" process $\mathrm{e}^{-}+\mathrm{p} \rightarrow v_{\mathrm{e}}+\mathrm{n}$, and are trapped for a few milliseconds in the outskirts of the core, just behind the shock. The prospects for detecting the prompt burst are not bright: The cross section for $\left(v_{e}\right)_{L}$ scattering is far lower than for $\left(\bar{v}_{e}\right)_{R}$ scattering. It is however conceivable that $\sim 1$ such neutrino would be detected ${ }^{\# 3}$. Intriguingly, the first event in Kamiokande seems to be a $\left(v_{\mathrm{e}}\right)_{\mathrm{L}}$ event, suggesting therefore that the prompt burst was indeed detected. (Neutrinos are detected through $\left(v_{\mathrm{e}}\right)_{\mathrm{L}}+\mathrm{e}^{-} \rightarrow\left(v_{\mathrm{e}}\right)_{\mathrm{L}}$ $+\mathrm{e}^{-}$scattering and antineutrinos through $\left(\bar{v}_{\mathrm{e}}\right)_{\mathrm{R}}+$ $\mathrm{p} \rightarrow \mathrm{e}^{+}+\mathrm{n}$ scattering. The outgoing electron in the first process preserves the information about the direction of motion of the incident particle while the positron in the second process completely loses it. The first Kamiokande event is very "directional" with respect to the Large Magelanic Cloud, hence the inclination to relate it to a $\left(v_{\mathrm{e}}\right)_{\mathrm{L}}$ event $)$. Suppose that the neutrinos are endowed with an LMA magnetic transition moment, and let us consider the implications for the prompt burst: It could be depleted by electromagnetic scattering in the core and by passing through resonances on the way out of the star. As mentioned before, not much is known about the resonances. We are therefore free to assume that no drastic (more than $50 \%$ ) depletion occurs there. As for electromagnetic scattering in the core - it induces $\left(v_{\mathrm{e}}\right)_{\mathrm{L}} \rightarrow\left(\bar{v}_{\mu}\right)_{\mathrm{R}}$ conversion at a rate [21]

$t^{-1} \simeq 4 \times 10^{24}\left(\mu / \mu_{\mathrm{B}}\right)^{2} Y_{\mathrm{e}}\left(\rho / \rho_{0}\right)\left(1+B_{\mathrm{F}}\right) \mathrm{s}^{-1}$,

where $Y_{\mathrm{e}}$ is the electron abundance, $\rho$ is the matter density, $\rho_{0}=2.7 \times 10^{14} \mathrm{~g} / \mathrm{cm}^{3}$ is the inner core density and $B_{\mathrm{F}} \sim \frac{1}{2}$ is the Pauli blocking factor, due to

\footnotetext{
\#2 The implications of the prompt neutronization burst for the MSW solution of the solar neutrino puzzle were studied in ref. [19].

\#3 According to a model by Arnett [20], the prompt neutronization burst carries more of the collapse energy and its duration is longer. It is then possible that the first two events in Kamiokande were $\left(v_{c}\right)_{L}$ events.
}

electron degeneracy. For $\mu \simeq 10^{-11} \mu_{\mathrm{B}}$ and $\rho \simeq 10^{-11}$ $\mathrm{g} / \mathrm{cm}^{3}$ (the density at the region where the prompt neutrinos are trapped)

$t^{-1} \leqslant 0.1 \mathrm{~s}^{-1}$.

Such a rate is far too slow to effect the prompt neutrinos that are trapped for only a few ms. We conclude that the LMA scenario is consistent also with the possibility that Kamiokande detected the prompt neutrino burst.

Another cosmological bound. Here we will consider the possibility that the dominant decay mode of $v_{\mu}$ is induced by the magnetic transition moment: $v_{\mu} \rightarrow \bar{v}_{\mathrm{e}} \gamma$. The $v_{\mu}$ lifetime is then $\tau\left(v_{\mu}\right)=\left[(1 / 8 \pi) \mu^{2} m^{3}\right]^{-1} \sim 10^{33} s$, where $m$ is the $v_{\mu}$ mass, assumed to be equal to $\sqrt{\Delta m^{2}} \sim 10^{-4} \mathrm{eV}$, and $\mu$ is $10^{-11} \mu_{\mathrm{B}}$. Cowsik [22] derived a bound on light neutrinos that decay mainly electromagnetically and live longer than the universe ( $\tau_{\text {universe }} \sim 10^{10} \mathrm{yr}$ ). His bound reads

$\tau / 1 \mathrm{~s} \geqslant 10^{27}(\mathrm{~m} / 1 \mathrm{eV})$.

Substituting the lifetime and mass of the electromagnetically decaying LMA neutrino we find Cowsik's bound is easily satisfied.

Finally, we would like to briefly discuss the Zeldovich-Konopinsky-Mahmoud (ZKM) neutrino [23]. The ZKM model has two basic ingredients: The neutrinos are of Majorana type and $L_{\mathrm{e}}-L_{\mu}$ is a conserved quantum number. ( $L_{\mathrm{e}}, L_{\mu}$ are electron and muon lepton numbers respectively). There is only one mass term which involves the $v_{\mathrm{e}}$ 's and $v_{\mu}$ 's:

$m\left(\vec{v}_{\mu}\right)_{\mathrm{R}}\left(v_{\mathrm{e}}\right)_{\mathrm{L}}+$ h.c.

It therefore becomes possible to think of $\left(V_{c}\right)_{L}$ and $\left(\bar{v}_{\mu}\right)_{R}$ as the left- and right-handed components of a single Dirac neutrino. This neutrino, being of Dirac type, may have a magnetic moment [24] $\mu \sim 10^{-11} \mu_{\mathrm{B}}$. It then solves the solar neutrino problem in the VVO way and avoids the supernova bound in the LMA way: In the sun, the ZKM neutrino undergoes a VVO-like spin precession (rather than passing through an LMA resonance), so that the original $\left(v_{e}\right)_{L}$ turns into the undetectable $\left(\bar{v}_{\mu}\right) R$. In the supernova core, electromagnetic scatterings on protons and electrons will convert $\left(v_{e}\right)_{L}$ 's into $\left(\bar{v}_{\mu}\right)_{R}$ 's. As in the LMA case, the final $\left(\bar{V}_{\mu}\right)_{R}$ 's are not sterile but weakly interacting and 
trapped in the core. Consequently, no new bound on the magnetic moment results from the observation of the SN 1987A neutrino pulse. Thus, the ZKM neutrino provides still another possible solution to the solar neutrino puzzle which incorporates anticorrelation of neutrino flux with sun spot activity and is consistent with all presently known bounds on the neutrino magnetic moment.

Summarizing, all bounds discussed here allow an LMA type solution of the solar neutrino problem with magnetic transition moment $\mu \sim 10^{-11} \mu_{\mathrm{B}}$ and mass squared difference $\Delta m^{2} \sim 10^{-8} \mathrm{eV}^{2}$. A ZKM neutrino with $\mu \sim 10^{-11} \mu_{\mathrm{B}}$ is also allowed. In particular, the observation of the 1987 supernova neutrino pulse, which seems to put the VVO solution in serious difficulties comfortably allows the LMA (or ZKM) scenario.

We would like to thank David Arnett, Josh Frieman, Dennis Hegyi and Lincoln Wolfenstein for useful communications.

\section{References}

[1] M.B. Voloshin and M.I. Vysotsky ITEP Report No. 1 (1986); L.B. Okun, Yad. Fiz. 44 (1986) 847 [Sov. J. Nucl. Phys. 44 (1986) 546];

L.B. Okun, M.B. Voloshin and M.I. Vysotsky, Yad. Fiz, 91 (1986) 754 [Sov. J. Nucl. Phys. 44 (1986) 440]; Zh. Exp. Theor. Fiz. 91 (1986) 754 [Sov. Phys. JETP 64 (1986) 446].

[2] A. Cisneros, Astrophys. Space Sci. 10 (1981) 87.

[3] G.A. Bazilevskaya, Yu.I. Stozhkov and T.N. Charahchyan, pis'ma Zh. Eksp. Theor. Fiz. 35 (1982) 273 [JETP Lett. 35 (1982) 341];

G.A. Bazilevskaya et al., Yad. Fiz. 39 (1984) 856 [Sov. J. Nucl. Phys. 39 (1984) 543];

R. Davis, in: Proc. Seventh Workshop on Grand unification (Toyama, Japan, 1986), ed. J. Arafune (World Scientific, Singapore, 1986).

[4] A.V. Kyuldjiev, Nucl. Phys. B 243 (1984) 387.

[5] J. Morgan, Phys. Lett. B 102 (1981) 247.
[6] P. Sutherland et al., Phys. Rev. D 13 ( 1976 ) 2700; M.A.B. Beg, W.J. Marciano and M. Ruderman, Phys. Rev. D 17 (1978) 1395;

M. Fukugita and S. Yazaki, Phys. Rev. D 36 (1987) 3817.

[7] S. Nussinov and Y. Rephaeli, Phys. Rev. D 36 (1987) 2278; I. Goldman, Y. Aharonov, G. Alexander and S. Nussinov, Phys. Rev. Lett. 60 (1988) 1789;

J.M. Lattimer and J. Cooperstein, Phys. Rev. Lett. 61 (1988) 23;

R. Barbieri and R.N. Mohapatra, Phys. Rev. Lett. 61 (1988) 27;

D. Notzold, Phys. Rev. D 38 (1988) 1658.

[8] C.S. Lim and W.J. Marciano, Phys. Rev. D 37 (1988) 1368.

[9] E.Kh. Akhmedov, preprint IAE-4568/1 (1988).

[10] P. Mikheyev and A.Yu. Smirnov, Nuovo Cimento C 9 (1986) 17.

[11] L. Wolfenstein, Phys. Rev. D 17 (1978) 2369

[12] See, e.g., J.R. Wilson et al., Ann. NY Acad. Sci. 470 (1986) 267;

R. Mayle, J.R. Wilson and D.N. Schramm, Fermilab preprint Fermilab 86/81-A (1986);

A. Burrows and J.M. Lattimer, Astron. J. 307 (1986) 178.

[13] A. Burrows and T.L. Mazurek, Nature 301 (1983) 315.

[14] M.B. Voloshin, Phys. Lett. B 209 (1988) 360.

[15] Kamiokande II Collab., Phys. Rev. Lett. 58 (1987) 1490; IMB Collab., Phys. Rev. Lett. 58 (1987) 1497.

[16] R. Barbieri, R.N. Mohapatra and T. Yanagida, University of Maryland preprint, UM PP \# 88-232.

[17] M. Fukugita and T. Yanagida, Phys. Rev. Lett. 58 (1987) 1807;

K.S. Babu and V.S. Mathur. Phys. Lett. B 196 (1987) 218.

[18] L. Wolfenstein, private communication.

[19] S.P. Mikheyev and A.Yu. Smirnov, JETP Lett. 46 (1987) 10 ;

J. Arafune, M. Fukugida, T. Yanagida and M. Yoshimura, Phys. Rev. Lett. 59 (1987); Phys. Lett. B 194 (1987) 477; T.K. Kuo and J. Pantaleone, Phys. Rev. D 37 (1988) 298; D. Notzold, Phys. Lett. B 196 (1987) 315.

[20] D. Arnett, Supernova theory and supernova 1987A (Shelton), to be published.

[21] H.A. Bethe, Proc. Camb. Philos. Soc. 31 (1935) 108; G.V. Domogatskii and D.K. Nadezhin, Sov, J. Nucl. 12 (1971) 678 .

[22] R. Cowsik. Phys. Rev. Lett. 39 (1977) 784.

[23] Ya.B. Zeldovich, Dok. Akad. Nauk SSSR 86 (1952) 505; E.J. Konopoinsky and J. Mahmoud, Phys. Rev. 92 (1953) 1045.

[24] L. Wolfenstein, Nucl. Phys. B 186 (1981) 147; S.T. Petcov, Phys. Lett. B 115 (1982) 401. 